IEE Proceedings D: Control Theory and Applications, 1992, IEEE, Vol. 139, Iss. 3, pp.

\title{
Performance in Sampled Data Systems
}

\author{
Control Systems Centre Report 733
}

\section{Abstract}

A performance index for control systems has been defined as the supremum of the absolute error that occurs over all time and all inputs. The input to the system is known only to the extent that it belongs to a known function space. This paper shows how this performance index is determined for certain sampled data systems with an input space characterised only by a bound on the derivative of the input. An example of a design using this performance index is given.

\section{J F Whidborne}

Control Systems Centre, University of Manchester Institute of Science and Technology, Sackville Street, Manchester M60 1QD.

Revised September 1991

September 1990 


\section{Introduction}

A measure of performance for control systems has been defined by Zakian [1]. The lack of performance $\hat{e}$ is defined as the least upper bound of the absolute error that occurs over all time and all inputs

$$
\hat{e}=\sup \{|e(t, f)|: t \in \mathbb{R}, f \in \mathcal{F}\}
$$

where $e(t, f)$ is the system error, $t$ is time and $f \in \mathcal{F}$ is the system input, which belongs to the known function space of inputs $\mathcal{F}$. Zakian [1] derived an expression to determine $\hat{e}$ for linear time-invariant analogue control systems where the input space $\mathcal{F}$ is characterised by a uniform bound on the derivative of the input. However, this expression is not applicable to sampled data control systems, this paper shows how $\hat{e}$ can be determined for certain sampled data control systems.

The calculation of $\hat{e}$ is less simple for sampled data systems than for continuous time systems. However it is relatively simple to calculate the lack of performance $\hat{e}^{*}$ at the sampling instances rather than over all time where

$$
\hat{e}^{*}=\sup \left\{\left|e^{*}(k, f)\right|: k=0,1,2, \ldots, \quad f^{*} \in \mathcal{F}^{*}\right\}
$$

where $e^{*}\left(k, f^{*}\right)$ is the system error at the sampling instances, $k$ is unit of discrete time and $f^{*} \in \mathcal{F}^{*}$ is the system input, which belongs to the known function space of inputs $\mathcal{F}^{*}$. An expression is derived in Section 2 which shows how $\hat{e}^{*}$ can be determined for any linear sampled data system. This expression is analogous to the expression derived by Zakian.

The error between the sampling instances may exceed the error at the sampling instances, in such cases $\hat{e}$ is greater than $\hat{e}^{*}$. Section 3 shows how $\hat{e}$ can be determined for the discrete time system shown in Fig. 1. The relationship between $\mathcal{F}$ and $\mathcal{F}^{*}$, and hence the relationship between $\hat{e}$ and $\hat{e}^{*}$ is given in Section 4

The performance index $\hat{e}$ is of particular value when applied to 'critical' control systems [2]. A critical control system is one where the error is required to be strictly within prescribed bounds, any violation of these bounds results in unacceptable operation. An example of the design of an analogue critical control system is given in [3]. Section 5 outlines a design procedure for critical sampled data control systems using $\hat{e}$ as the performance index, and Section 6 shows the design of a sampled data control system for the pointing mechanism of a satellite earth scanning antenna. 


\section{Performance at Sampling Points}

The input $f^{*}(k)$ is known only to the extent that it belongs to the known function space $\mathcal{F}^{*}$ which is defined as the class of all functions $f^{*}: \mathbb{Z} \rightarrow \mathbb{R}$ such that

$$
f^{*}(k)=0 \quad \forall \quad k=-1,-2, \ldots, \quad f^{*} \in \mathcal{F}^{*}
$$

and there is a finite positive number $D^{*}$ such that

$$
D^{*}=\sup \left\{\left|f^{*}(k)-f^{*}(k-1)\right|: k=0,1,2, \ldots, \quad f^{*} \in \mathcal{F}^{*}\right\}
$$

The response of the error at the $k$ th unit of discrete time to the input $f^{*}$ is denoted by $e^{*}\left(k, f^{*}\right)$.

For all $f^{*} \in \mathcal{F}^{*}$ the output is related to the input by the convolution summation

$$
e^{*}\left(k, f^{*}\right)=\sum_{n=0}^{k} e^{*}(k-n) f^{*}(n)
$$

The response $e^{*}(k-n)$ is simply the weighting sequence $e^{*}(k)$ delayed $n$ units of discrete time, where $e^{*}(k)$ is the response to the Kronecker delta sequence applied at $k=0$. The unit step response $e^{*}(k, h)$ is the response to the unit step $h(k)$ where

$$
h(k)= \begin{cases}0, & k<0 \\ 1, & k \geq 0\end{cases}
$$

Thus

$$
e^{*}(k, h)=\sum_{n=0}^{k} e^{*}(k-n)=\sum_{n=0}^{k} e^{*}(n)
$$

so the input output rule can be expressed as

$$
\begin{aligned}
e^{*}\left(k, f^{*}\right) & =\sum_{n=0}^{k} e^{*}(k-n) f^{*}(n) \\
& =\sum_{n=0}^{k}\left\{\sum_{i=0}^{k-n} e^{*}(i)-\sum_{i=0}^{k-n-1} e^{*}(i)\right\} f^{*}(n) \\
& =\sum_{n=0}^{k} e^{*}(k-n, h) f^{*}(n)-e^{*}(k-n-1, h) f^{*}(n) \\
& =\sum_{n=0}^{k} e^{*}(k-n, h)\left[f^{*}(n)-f^{*}(n-1)\right]
\end{aligned}
$$

A bound $\hat{e}^{*}$ on the error is now stated as

$$
\hat{e}^{*}=\sup \left\{\left|e^{*}\left(k, f^{*}\right)\right|: k=0,1,2, \ldots, \quad f^{*} \in \mathcal{F}^{*}\right\}
$$




\section{Performance at Sampling Points}

The input $f^{*}(k)$ is known only to the extent that it belongs to the known function space $\mathcal{F}^{*}$ which is defined as the class of all functions $f^{*}: \mathbb{Z} \rightarrow \mathbb{R}$ such that

$$
f^{*}(k)=0 \quad \forall \quad k=-1,-2, \ldots, \quad f^{*} \in \mathcal{F}^{*}
$$

and there is a finite positive number $D^{*}$ such that

$$
D^{*}=\sup \left\{\left|f^{*}(k)-f^{*}(k-1)\right|: k=0,1,2, \ldots, \quad f^{*} \in \mathcal{F}^{*}\right\}
$$

The response of the error at the $k$ th unit of discrete time to the input $f^{*}$ is denoted by $e^{*}\left(k, f^{*}\right)$.

For all $f^{*} \in \mathcal{F}^{*}$ the output is related to the input by the convolution summation

$$
e^{*}\left(k, f^{*}\right)=\sum_{n=0}^{k} e^{*}(k-n) f^{*}(n)
$$

The response $e^{*}(k-n)$ is simply the weighting sequence $e^{*}(k)$ delayed $n$ units of discrete time, where $e^{*}(k)$ is the response to the Kronecker delta sequence applied at $k=0$. The unit step response $e^{*}(k, h)$ is the response to the unit step $h(k)$ where

$$
h(k)= \begin{cases}0, & k<0 \\ 1, & k \geq 0\end{cases}
$$

Thus

$$
e^{*}(k, h)=\sum_{n=0}^{k} e^{*}(k-n)=\sum_{n=0}^{k} e^{*}(n)
$$

so the input output rule can be expressed as

$$
\begin{aligned}
e^{*}\left(k, f^{*}\right) & =\sum_{n=0}^{k} e^{*}(k-n) f^{*}(n) \\
& =\sum_{n=0}^{k}\left\{\sum_{i=0}^{k-n} e^{*}(i)-\sum_{i=0}^{k-n-1} e^{*}(i)\right\} f^{*}(n) \\
& =\sum_{n=0}^{k} e^{*}(k-n, h) f^{*}(n)-e^{*}(k-n-1, h) f^{*}(n) \\
& =\sum_{n=0}^{k} e^{*}(k-n, h)\left[f^{*}(n)-f^{*}(n-1)\right]
\end{aligned}
$$

A bound $\hat{e}^{*}$ on the error is now stated as

$$
\hat{e}^{*}=\sup \left\{\left|e^{*}\left(k, f^{*}\right)\right|: k=0,1,2, \ldots, \quad f^{*} \in \mathcal{F}^{*}\right\}
$$


$\hat{e}^{*}$ is the greatest error as $k$ ranges over the space $\mathbb{Z}$ and the input ranges over the input space $\mathcal{F}^{*}$.

\section{Theorem 1}

The following result

$$
\hat{e}^{*}=D^{*}\left\|e^{*}(h)\right\|_{1}
$$

provides a simple formula for computing $\hat{e}^{*}$ where

$$
\left\|e^{*}(h)\right\|_{1}=\sum_{n=0}^{\infty}\left|e^{*}(n, h)\right|
$$

Proof

Now

$$
\hat{e}^{*}=\sup \left\{\left|e^{*}\left(k, f^{*}\right)\right|: k=0,1,2, \ldots, \quad f^{*} \in \mathcal{F}^{*}\right\}
$$

Since $f^{*}(k)=0$ for $k=-1,-2, \ldots$, it follows that, from (11)

$$
\begin{gathered}
\left|e^{*}\left(k, f^{*}\right)\right| \leq \sum_{n=0}^{k}\left|e^{*}(k-n, h)\right|\left|f^{*}(n)-f^{*}(n-1)\right| \\
\sup _{f^{*} \in \mathcal{F}^{*}}\left|e^{*}\left(k, f^{*}\right)\right| \leq D^{*} \sum_{n=0}^{k}\left|e^{*}(k-n, h)\right| \\
\sup _{f^{*} \in \mathcal{F}^{*}} \sup _{k \in \mathbb{Z}}\left|e^{*}\left(k, f^{*}\right)\right| \leq D^{*} \sum_{n=0}^{\infty}\left|e^{*}(n, h)\right| \\
\hat{e}^{*} \leq D^{*}\left\|e^{*}(h)\right\|_{1}
\end{gathered}
$$

Now consider an input sequence $f_{w}^{*} \in \mathcal{F}^{*}$ where

$$
f_{w}^{*}(n)-f_{w}^{*}(n-1)= \begin{cases}D^{*} \operatorname{sgn} e^{*}(k-n), & 0 \leq n \leq k \\ 0, & n<0\end{cases}
$$

which from (11) gives

$$
\begin{gathered}
e^{*}\left(k, f_{w}^{*}\right)=D^{*} \sum_{n=0}^{k}\left|e^{*}(k-n, h)\right| \\
=D^{*} \sum_{n=0}^{k}\left|e^{*}(n, h)\right| \\
\sup _{k \in \mathbb{Z}}\left|e^{*}\left(k, f_{w}^{*}\right)\right| \geq e^{*}\left(k, f_{w}^{*}\right)
\end{gathered}
$$

thus

$$
\hat{e}^{*} \geq D^{*}\left\|e^{*}(h)\right\|_{1}
$$


So from (19) and (24)

$$
\hat{e}^{*}=D^{*}\left\|e^{*}(h)\right\|_{1}
$$

and the theorem is proved.

This theorem is analogous to that for the continuous time case given by Zakian [1].

\section{Performance Over All Time}

The limitation of theorem 1 is that it gives the supremum of the output at the sampling points only. This value may be exceeded at times between the sampling points, so a means of calculating the supremum of the error at all times $\hat{e}$, as defined in (1) is required.

Therefore the system is considered as a continuous time system, with continuous inputs and outputs. However, the process of sampling means that a digital control scheme which is composed of linear time-invariant (LTI) components and fixed parameter digital controllers is a linear time-variant (LTV) system when considered as a continuous time system.

Consider the system shown in Fig. 2. An input which occurs at a time between samples will not be seen by the plant until the next sample, so the plant cannot respond until the sampling instant. The relationship between the system input and output is thus not independent of time, therefore the system cannot be timeinvariant.

For an LTV system, the error at any time is related to the input by the linear time-variant convolution

$$
e(t, f)=\int_{0}^{t} e(t, \tau, \delta) f(\tau) d \tau
$$

where $f(t)$ is the input and $e(t, \tau, \delta)$ is the response at time $t$ to a unit impulse $\delta(t-\tau)$ applied at time $\tau$.

Thus in order be able to calculate $\hat{e}$, an expression which gives the supremum of the error at any particular time for all LTV systems is first derived. From this, an expression from which $\hat{e}$ can be calculated is derived for the particular sampled data control scheme shown in Figure 1.

The input is known to the extent that it belongs to the known function space $\mathcal{F}$ which is defined as the set of all functions $f: \mathbb{R} \rightarrow \mathbb{R}$ such that $f(t)=0$ for $t \leq 0$, 
the derivative $f^{(1)}$ is piecewise continuous and there is a finite positive number $D$ such that

$$
D=\sup \left\{\left|f^{(1)}(t)\right|: t \geq 0, f \in \mathcal{F}\right\}
$$

Let $e(t, \tau, h)$ denote the response at time $t$ to a unit step $h(t-\tau)$ where $h(t-\tau)=0$ for $t \leq \tau$ and $h(t-\tau)=1$ for $t>\tau$. From (26)

$$
e(t, \tau, h)=\int_{\tau}^{t} e\left(t, \tau^{\prime}, \delta\right) \mathrm{d} \tau^{\prime}
$$

which can be expressed as

$$
\begin{aligned}
e(t, \tau, h) & =\int_{0}^{t} e\left(t, \tau^{\prime}, \delta\right) \mathrm{d} \tau^{\prime}-\int_{0}^{\tau} e\left(t, \tau^{\prime}, \delta\right) \mathrm{d} \tau^{\prime} \\
& =e(t, 0, h)-\int_{0}^{\tau} e\left(t, \tau^{\prime}, \delta\right) \mathrm{d} \tau^{\prime}
\end{aligned}
$$

and differentiating gives

$$
\frac{\mathrm{d} e(t, \tau, h)}{\mathrm{d} \tau}=-e(t, \tau, \delta)
$$

Integrating (26) by parts and substituting (31) gives

$$
e(t, f)=-\left.e(t, \tau, h) f(\tau)\right|_{0} ^{t}+\int_{0}^{t} e(t, \tau, h) f^{(1)}(\tau) \mathrm{d} \tau
$$

and since $f(0)=0$ and $e(t, t, h)=0$,

$$
e(t, f)=\int_{0}^{t} e(t, \tau, h) f^{(1)}(\tau) \mathrm{d} \tau
$$

\section{Theorem 2}

The supremum of the error of a causal linear time-variant system at a specific time $t$ is given by

$$
\sup _{f \in \mathcal{F}}|e(t, f)|=D \int_{0}^{t}|e(t, \tau, h)| \mathrm{d} \tau
$$

Proof

From (33) and (27)

$$
\sup _{f \in \mathcal{F}}|e(t, f)| \leq D \int_{0}^{t}|e(t, \tau, h)| \mathrm{d} \tau
$$

For a time $t \geq 0$ there exists an $f_{w} \in \mathcal{F}$ defined by

$$
f_{w}^{(1)}(\tau)=\left\{\begin{array}{lll}
D \operatorname{sgn} e(t, \tau, h) & \text { for } & 0 \leq \tau \leq t \\
0 & \text { for } & \tau<0
\end{array}\right.
$$


which from (33) gives

$$
\begin{gathered}
e\left(t, f_{w}\right)=\int_{0}^{t} e(t, \tau, h) \operatorname{sgn} e(t, \tau, h) \mathrm{d} \tau \\
e\left(t, f_{w}\right)=D \int_{0}^{t}|e(t, \tau, h)| \mathrm{d} \tau
\end{gathered}
$$

So from (35) and (38), the theorem is proved.

\section{Theorem 3}

For the digital control scheme shown in Fig. 1 , for all $k=0,1,2, \ldots$ and $f \in \mathcal{F}$ with a given sampling interval $T$, the supremum of the error at the particular times $k T+t_{s}$ where $0 \leq t_{s}<T$, namely

$$
\tilde{e}\left(t_{s}\right)=\sup \left\{\left|e\left(k T+t_{s}, f\right)\right|: k=1,2, \ldots, \quad f \in \mathcal{F}\right\}
$$

is given by

$$
\tilde{e}\left(t_{s}\right)=D\left(t_{s}+T \sum_{k=0}^{\infty}\left|e\left(k T+t_{s}, 0, h\right)\right|\right)
$$

Proof

For the control scheme in Fig. 1, because the input is sampled, $e(t, \tau, h)$ remains constant at a time $t$ for the starting time $\tau$ ranging between sampling points. In addition, the initial error is unity, and remains so until the next sampling instant. A typical error step response is shown in Fig. 3.

So, for $k T \leq \tau<(k+1) T$,

$$
e(t, \tau, h)=\left\{\begin{array}{lll}
0 & \text { for } \quad t \leq \tau \\
1 & \text { for } \quad \tau<t \leq(k+1) T \\
e(t, k T, h) & \text { for } \quad t>(k+1) T
\end{array}\right.
$$

where $k=0,1,2, \ldots$ Let $n T<t \leq(n+1) T$, and so from (34)

$$
\sup _{f \in \mathcal{F}}|e(t, f)|=D\left(t_{s}+T \sum_{k=0}^{n}|e(t, k T, h)|\right)
$$

where $n=0,1,2, \ldots$ Let $t=n T+t_{s}$, then

$$
\sup _{f \in \mathcal{F}}\left|e\left(n T+t_{s}, f\right)\right|=D\left(t_{s}+T \sum_{k=0}^{n}\left|e\left(n T+t_{s}, k T, h\right)\right|\right)
$$

Because of the periodic nature of the system

$$
e(t, \tau, h)=e(t+k T, \tau+k T, h), \quad k=0,1,2, \ldots
$$


and so from (43)

$$
\sup _{f \in \mathcal{F}}\left|e\left(n T+t_{s}, f\right)\right|=D\left(t_{s}+T \sum_{k=0}^{n}\left|e\left(k T+t_{s}, 0, h\right)\right|\right)
$$

so for any $t_{s}$ over all sampling instances.

$$
\sup _{n=0,1,2, \ldots} \sup _{f \in \mathcal{F}}\left|e\left(n T+t_{s}, f\right)\right|=D\left(t_{s}+T \sum_{k=0}^{\infty}\left|e\left(k T+t_{s}, 0, h\right)\right|\right)
$$

and so the theorem is proved.

From theorem 3, it is easy to determine $\hat{e}$ numerically by a one-dimension search for the maximum value of $\tilde{e}$ over $t_{s}$, so

$$
\hat{e}=\sup _{0 \leq t_{s}<T} \tilde{e}\left(t_{s}\right)
$$

\section{The Relationship Between $\mathcal{F}$ and $\mathcal{F}^{*}$}

The input $f^{*}(k) \in \mathcal{F}^{*}$ represents a periodic sample of an input $f(t) \in \mathcal{F}$. If the sampling period is $T$, then from (4) and (27) then

$$
D^{*}=D T
$$

Thus from (40) for $t_{s}=0$, (i.e. at the sampling instant)

$$
\tilde{e}(0)=D T \sum_{k=0}^{\infty}|e(k T, 0, h)|
$$

which is equivalent to (13)

\section{Design Procedure}

The method of inequalities $[4,5]$ is a multi-objective formulation of the design problem expressed as

$$
\phi_{i}(p) \leq \varepsilon_{i} \quad \text { for } \quad i=1 \ldots n
$$

where $\phi_{i}$ are performance indices, $p \in \mathbb{R}^{N}$ is the set of design parameters, and $\varepsilon_{i}$ are real numbers chosen by the designer representing tolerable values of the performance 
indices. The aim is the satisfaction of the set of inequalities in order that an acceptable design is reached. The inequality (50) may be solved by numerical methods such as the moving boundaries process (MBP) [4].

The performance measure $\hat{e}(p)$ can be used in conjunction with the method of inequalities by setting $\phi_{1}(p)=\hat{e}(p)$ where

$$
\hat{e}(p)=\sup \{|e(t, f, p)|: t \in \mathbb{R}, f \in \mathcal{F}\}
$$

with $p$ representing the parameters of the controller $K(z, p)$. The problem is thus to satisfy

$$
\hat{e}(p) \leq \varepsilon
$$

where $\varepsilon$ is the maximum tolerable value of the system error $e$. Additional performance indices, such as the maximum control effort or the bandwidth, could be included if desired.

Expression (47) is more computationally expensive than (13). Therefore, to reduce the computational effort, the design procedure is conducted in two stages:

i) Use the MBP to solve the amended design problem

$$
\hat{e}^{*}(p) \leq \varepsilon
$$

where

$$
\hat{e}^{*}(p)=\sup \left\{\left|e^{*}\left(k, f^{*}, p\right)\right|: k=0,1,2, \ldots, \quad f^{*} \in \mathcal{F}^{*}\right\}
$$

using (13). Calculate $\hat{e}(p)$, and if $\hat{e}(p)>\varepsilon$ then go on to the second stage.

ii) Solve the design problem (52) using the MBP in conjunction with (47).

It should be noted that, from $(41), e(T, 0, h)=1$. So, from (49)

$$
\tilde{e}(0) \geq D T
$$

and hence from (47)

$$
\hat{e} \geq D T
$$

This means that $\hat{e}$ cannot be made less than $D \times T$. It also gives a means of choosing the sampling period $T$, from (56) and (52)

$$
T \leq \frac{\varepsilon}{D}
$$




\section{Design of an Earth Scanning Satellite Antenna Control System}

An earth scanning antenna on a space satellite is to be used to collect information on temperature and humidity using sensors which pick up microwave radiation emitted naturally from the earth and its atmosphere. The antenna is required to follow a variety of position reference profiles which have a maximum reference velocity of $1.0 \mathrm{rad} / \mathrm{sec}$. The design requirement is that the positional error be less than 1.2 degrees. To keep down hardware costs, a microprocessor based servo control system is used to drive the pointing mechanism.

Figure 4 shows a block diagram of a linear model of the servo system dynamics, where:

$u(s) \quad$ Voltage applied across DC servo motor

$v_{e}(s) \quad$ Net voltage across motor armature

$i_{a}(s) \quad$ Current in motor armature

$\tau_{m}(s)$ Torque generated by motor at motor shaft

$\omega(s) \quad$ Angular velocity of motor shaft

$y(s) \quad$ Angular position of antenna

$v_{b}(s) \quad$ Back EMF generated by the motor

$K_{t} \quad$ Motor torque coefficient

$K_{v} \quad$ Motor back EMF constant (incorporating effect of viscous damping)

$R \quad$ Resistance of the motor armature winding

$L \quad$ Inductance of the motor armature winding

$J \quad$ Total mechanism inertia referred to the motor shaft output

$N \quad$ Gearbox reduction

The system dynamics are combined into a transfer function $G(s)$, which relates the angular displacement of the motor output shaft to the voltage applied at the motor. The antenna is assumed stiff, and non-linearities such as stiction and gearbox backlash, are ignored.

$$
G(s)=\frac{K_{t} / N}{s\left(J L s^{2}+J R s+K_{t} K_{v}\right)}
$$

Voltage to the motor is applied by a digital controller $K(z, p)$ via a zero-order hold unit. Input to the controller consists of the error $e$ between the reference position profile signal $f$ and the antenna positional output $y$. It is assumed that both $f(t)$ and $y(t)$ are scaled in radians. The reference signals of the position profiles are obtained from look-up tables, and the angular position of the antenna is measured 
by an inductosyn, which is sampled by the digital controller with a sampling period $T$. The discretised reference input profiles have previously been sampled from the desired continuous time profiles. Quantitisation effects of the signal measurements are ignored. The control scheme is thus in the form of Fig. 1.

The position reference profiles have a maximum reference velocity of $1.0 \mathrm{rad} / \mathrm{sec}$, so the input space bound $D$ from expression (27) is $D=1.0$. The input space bound $D^{*}$ is determined from (48). The design requirement is

$$
\varepsilon=1.2 \mathrm{deg}=0.0209 \mathrm{rad}
$$

The model parameters have the following values:

$$
\begin{aligned}
& K_{t}=0.19 \mathrm{Nm} / \mathrm{Amp} \\
& K_{v}=0.22 \mathrm{Vs} / \mathrm{rad} \\
& R=2.00 \mathrm{Ohms} \\
& L=1.40 \mathrm{H} \\
& J=7 \times 10^{-4} \mathrm{kgm}^{2} \\
& N=
\end{aligned}
$$

so the plant transfer function $G(s)$ in combination with the zero-order hold unit may be represented by the discrete transfer function

$$
G(z)=\mathcal{Z}\left[\frac{1-\exp (T s)}{s} G(s)\right]
$$

From (57) and (59)

$$
T \leq 0.0209 \mathrm{sec}
$$

Thus the design was initially tried with a sampling rate $T=20 \mathrm{msec}$. This was found to give unsatisfactory results, although (53) was satisfied, the MBP was unable to satisfy (52). Hence the design was repeated with $T=10 \mathrm{msec}$ and $T=5$ msec, both of which gave acceptable results with (52) satisfied. For $T=5 \mathrm{msec}$, this was achieved with the initial design. The results are shown in Table 1 . The step responses $e(0, t, h)$ with the sampling points of the final designs are shown in Figs. 5 to 7. Plots of $\tilde{e}\left(t_{s}\right)$ against $t_{s}$ of the final designs are shown in Figs. 8 to 10 .

\section{Concluding Remarks}

The performance measure $\hat{e}$ is a natural indicator of the performance of a control system which explicitly considers the system inputs. This performance measure is particularly suitable for the design of critical control systems. This paper extends 


\begin{tabular}{|l|c|c|c|c|}
\hline \multicolumn{2}{|c|}{} & $T=20 \mathrm{msec}$ & $T=10 \mathrm{msec}$ & $T=5 \mathrm{msec}$ \\
\hline \multirow{3}{*}{$\begin{array}{l}\text { Design } \\
\text { Stage (i) }\end{array}$} & $K(z)$ & $325 \frac{z^{2}-0.064 z-0.260}{z^{2}+1.415 z+0.448}$ & $703 \frac{z^{2}+0.136 z-0.498}{z^{2}+1.100 z+0.436}$ & $600 \frac{z^{2}-0.642 z-0.150}{z^{2}+0.330 z-0.068}$ \\
\cline { 2 - 5 } & $\hat{e}^{*}$ & $0.0206 \mathrm{rad}$ & $0.0209 \mathrm{rad}$ & $0.0205 \mathrm{rad}$ \\
\cline { 2 - 5 } & $\hat{e}$ & $0.1751 \mathrm{rad}$ & $0.0212 \mathrm{rad}$ & $0.0205 \mathrm{rad}$ \\
\hline \multirow{3}{*}{$\begin{array}{l}\text { Design } \\
\text { Stage (ii) }\end{array}$} & $K(z)$ & $177 \frac{z^{2}+0.295 z-0.455}{z^{2}+1.387 z+0.461}$ & $703 \frac{z^{2}+0.136 z-0.498}{z^{2}+1.210 z+0.436}$ & as above \\
\cline { 2 - 5 } & $\hat{e}$ & $0.0297 \mathrm{rad}$ & $0.0201 \mathrm{rad}$ & $0.0205 \mathrm{rad}$ \\
\hline
\end{tabular}

Table 1: Design Results

the results of Zakian [1] to certain sampled data systems, to provide a means of calculating $\hat{e}$. The design example shows that it is important to consider the performance at all time, particularly when the sampling time is comparatively long. As would be expected, the difference between $\hat{e}$ and $\hat{e}^{*}$ decreases with decreasing sampling period, as the system controller becomes closer to a continuous time controller.

The use of $\hat{e}^{*}$ to design sampled data control systems is closely related to the $l^{1}$ optimal control approach [6,7]. However, the $l^{1}$-optimisation approach does not consider intersample behaviour. Morari and Zafiriou [8] detail how sampled data control systems can be designed using $H^{\infty}$ and $H_{2}$ techniques which consider performance over all time. Additional analysis of intersample behaviour using $H^{\infty}$ and $\mathrm{H}_{2}$ techniques can be found in [9] and [10].

\section{Acknowledgements}

The author is grateful to Mr. P. Lane for his help and to Dr. V. Zakian for his advice and guidance.

\section{References}

[1] ZAKIAN, V.: 'A performance criterion', Int. J. Control, 1986, 43, (3), pp. 921-931. 
[2] ZAKIAN, V.: 'Critical systems and tolerable inputs', Int. J. Control, 1989, 49, (4), pp. 1285-1289.

[3] WHIDBORNE, J.F.: 'The design of the electo-magnetic suspension control system for a maglev vehicle - A critical system'. Control Systems Centre Report 710, UMIST, Manchester, 1988.

[4] ZAKIAN, V., and AL-NAIB, U.: 'Design of Dynamical and Control Systems by the Method of Inequalities', Proc. IEE, 1973, 120, (11), pp. 1421-1427.

[5] MACIEJOWSKI, J.M.: 'Multivariable Feedback Design', (Addison-Wesley, 1989)

[6] DAHLEH, M.A., and PEARSON, J.B.: ' $l^{1}$ - optimal feedback controllers for discrete-time systems', Proc. ACC, Seattle, USA, 1986.

[7] DAHLEH, M.A., and PEARSON, J.B.: ' $l^{1}$ - optimal controllers for MIMO discrete-time systems', IEEE Trans., 1987, AC-32, (4), pp. 314-322.

[8] MORARI, M., and ZAFIRIOU, E.: 'Robust Process Control' (Prentice-Hall, 1989)

[9] BAMIEH, B., PEARSON, J.B., FRANCIS, B.A., and TANNENBAUM, A.: 'A lifting technique for linear periodic systems with applications to sampleddata control', Syst. Control Lett., 1991, 17, pp. 79-88.

[10] LEUNG, G.M.H., PERRY, T.P., and FRANCIS, B.A.: 'Performance analysis of sampled-data control systems', Automatica, 1991, 27, (4), pp. 699-704. 
Fig. 1 : Sampled Data Control System Configuration

Fig. 2 : A Sampled Data System

Fig. 3 : Typical Error Step Response for the Sampled Data System

Fig. 4 : Linear Model of Antenna Servo

Fig. 5 : Step Response of Design Showing Sampling Points for $T=20 \mathrm{msec}$

Fig. 6 : Step Response of Design Showing Sampling Points for $T=10 \mathrm{msec}$

Fig. 7 : Step Response of Design Showing Sampling Points for $T=5 \mathrm{msec}$

Fig. $8: \tilde{e}\left(t_{s}\right)$ against $t_{s}$ for $T=20 \mathrm{msec}$

Fig. $9: \tilde{e}\left(t_{s}\right)$ against $t_{s}$ for $T=10 \mathrm{msec}$

Fig. $10: \tilde{e}\left(t_{s}\right)$ against $t_{s}$ for $T=5 \mathrm{msec}$ 


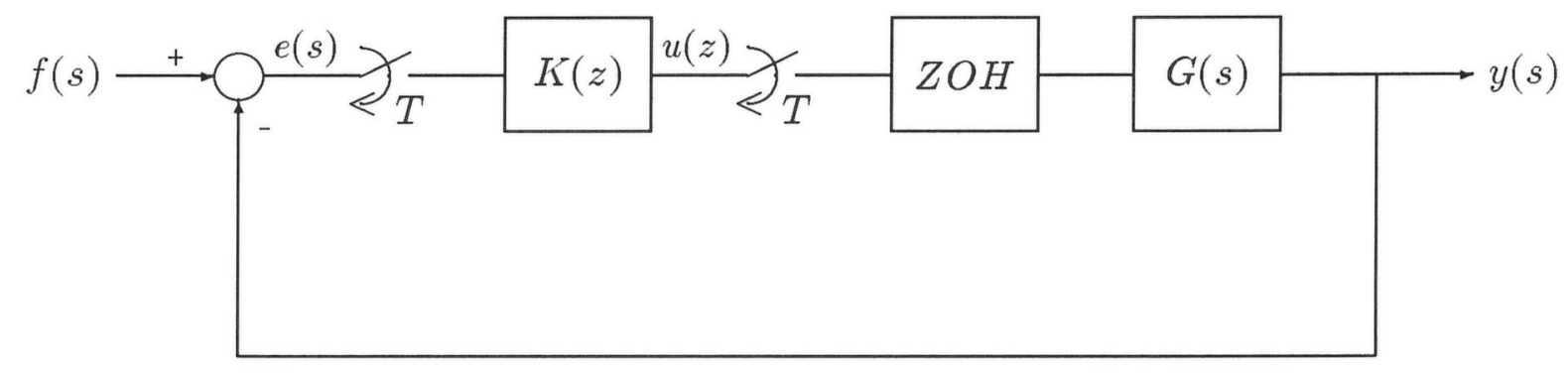

Figure 1: Sampled Data Control System Configuration 


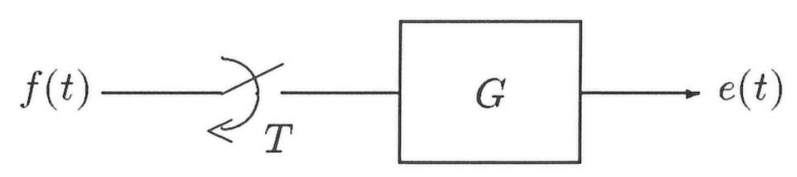

Figure 2: A Sampled Data System 


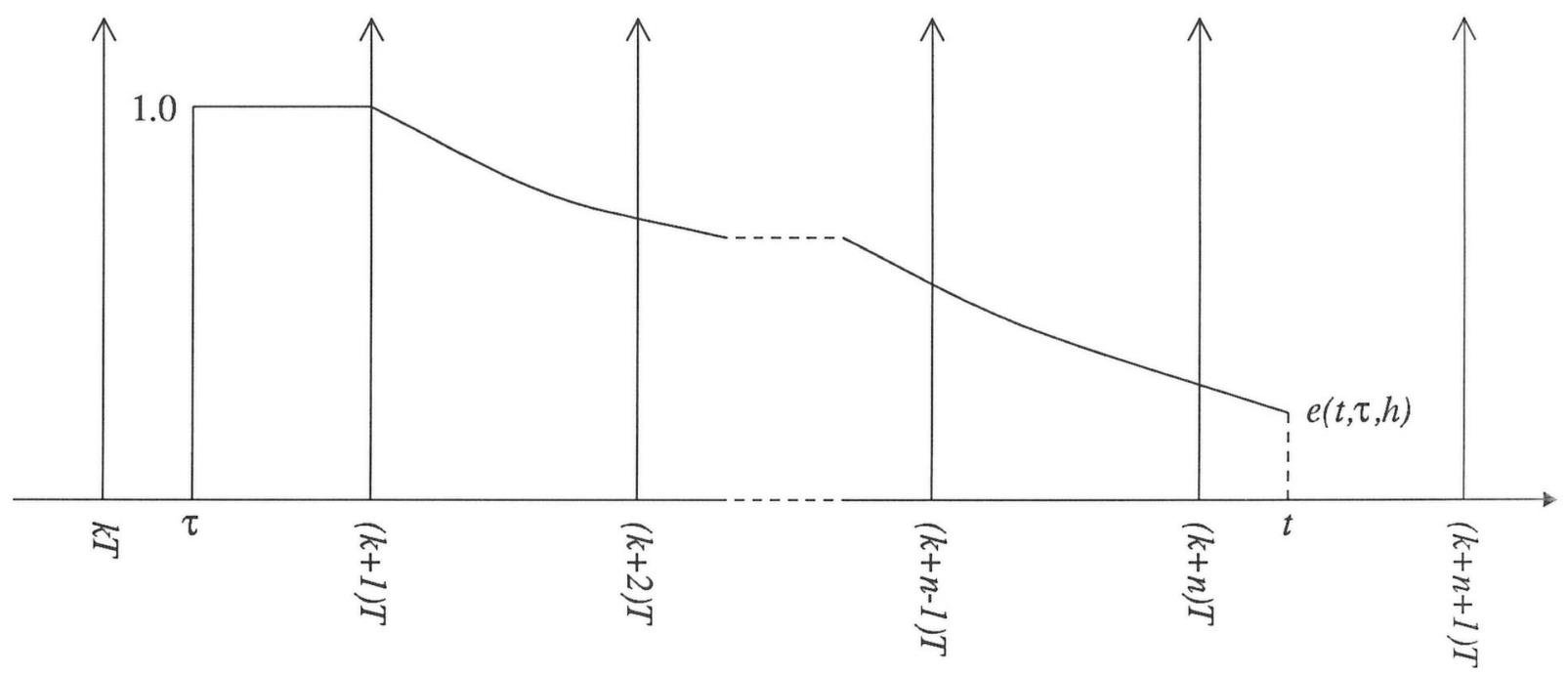

Figure 3: Typical Error Step Response for the Sampled Data System 


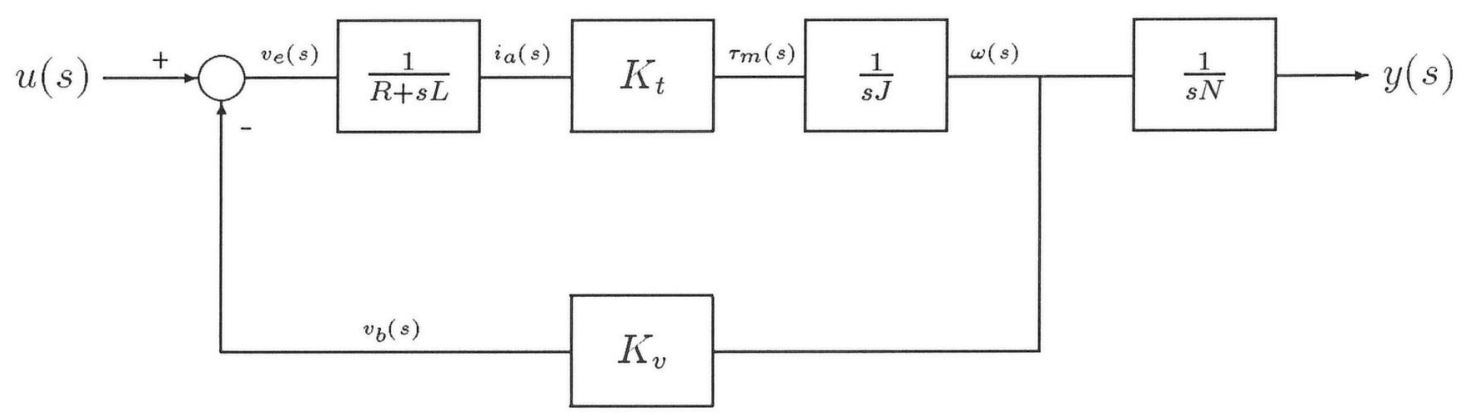

Figure 4: Linear Model of Antenna Servo 


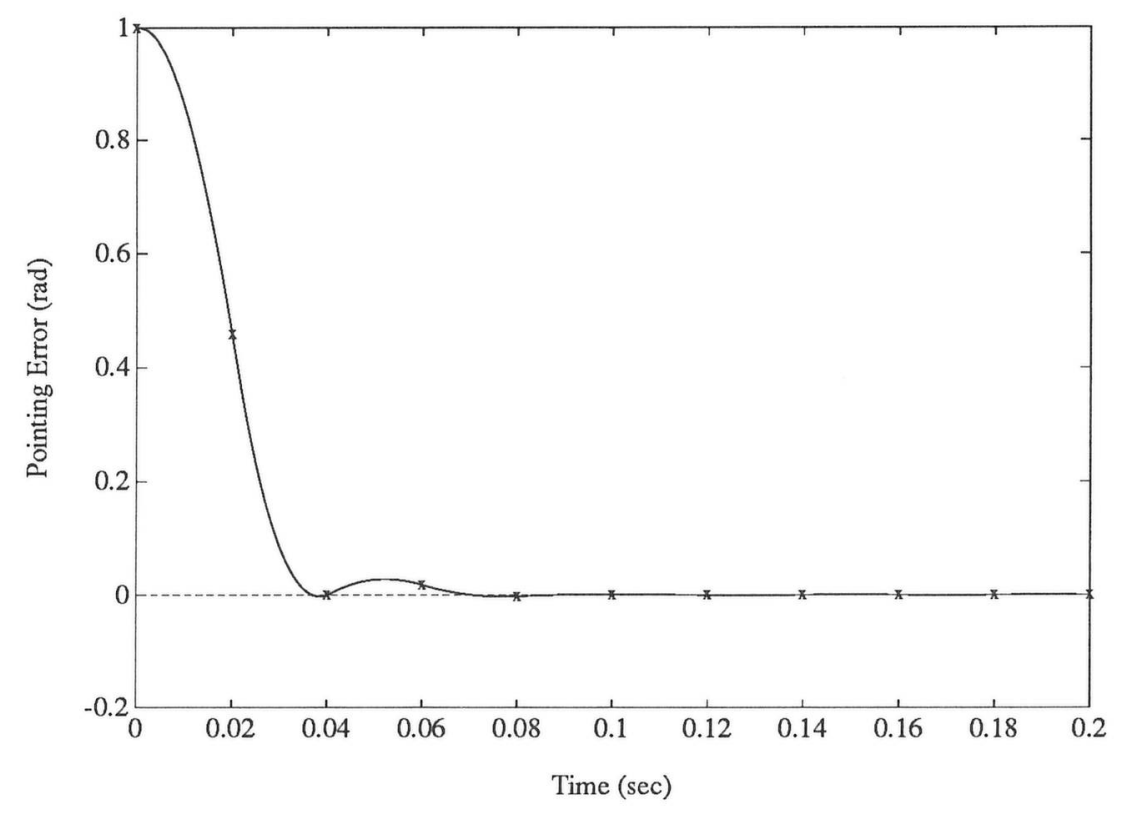

Figure 5: Step Response of Design Showing Sampling Points for $T=20 \mathrm{msec}$ 


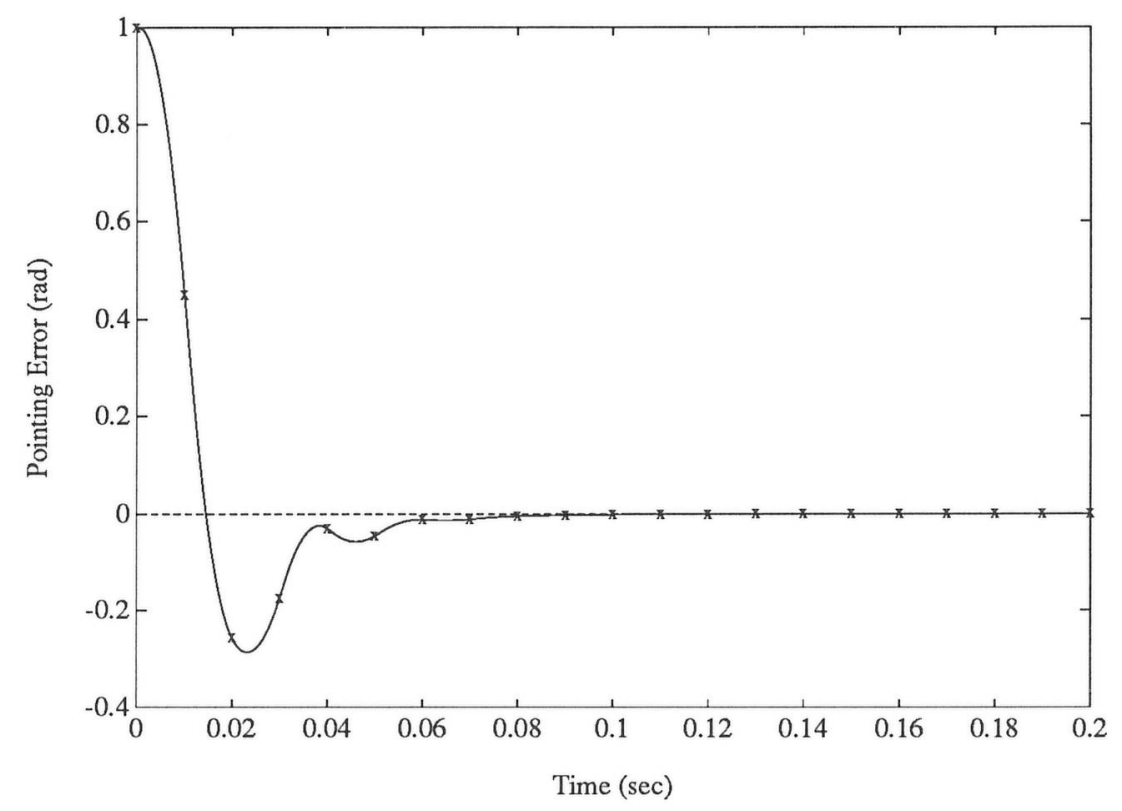

Figure 6: Step Response of Design Showing Sampling Points for $T=10 \mathrm{msec}$ 


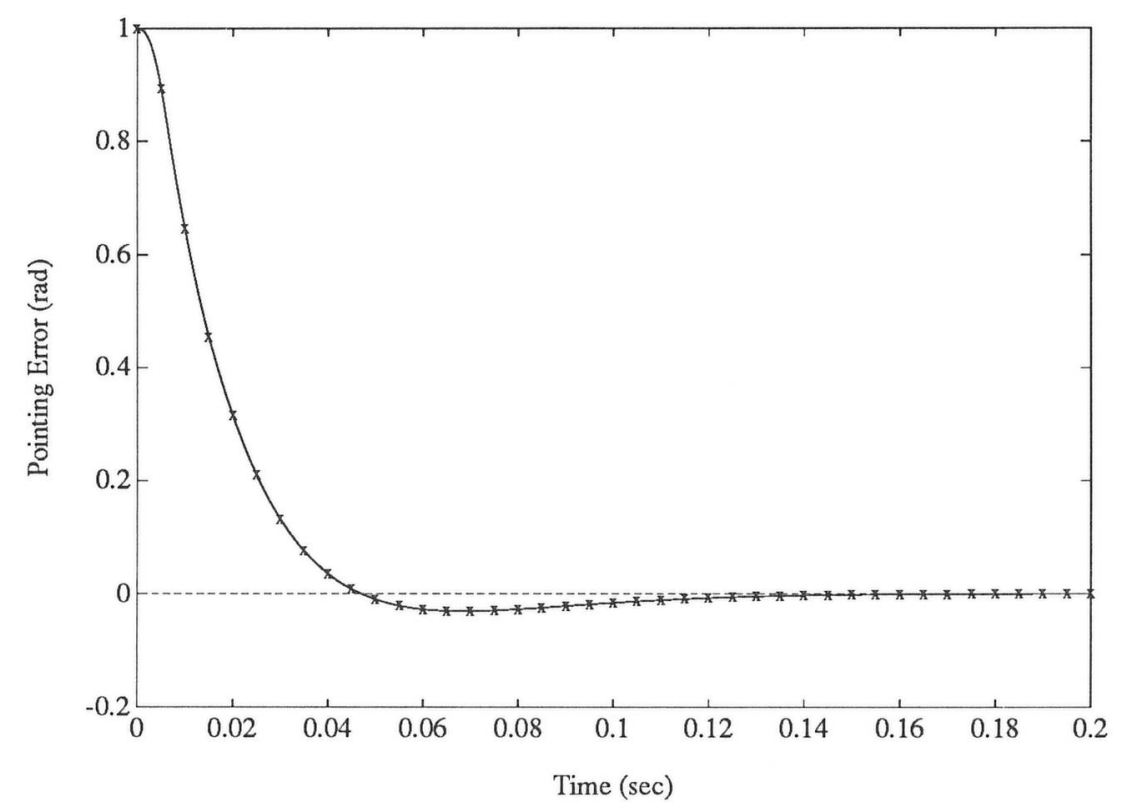

Figure 7: Step Response of Design Showing Sampling Points for $T=5 \mathrm{msec}$ 


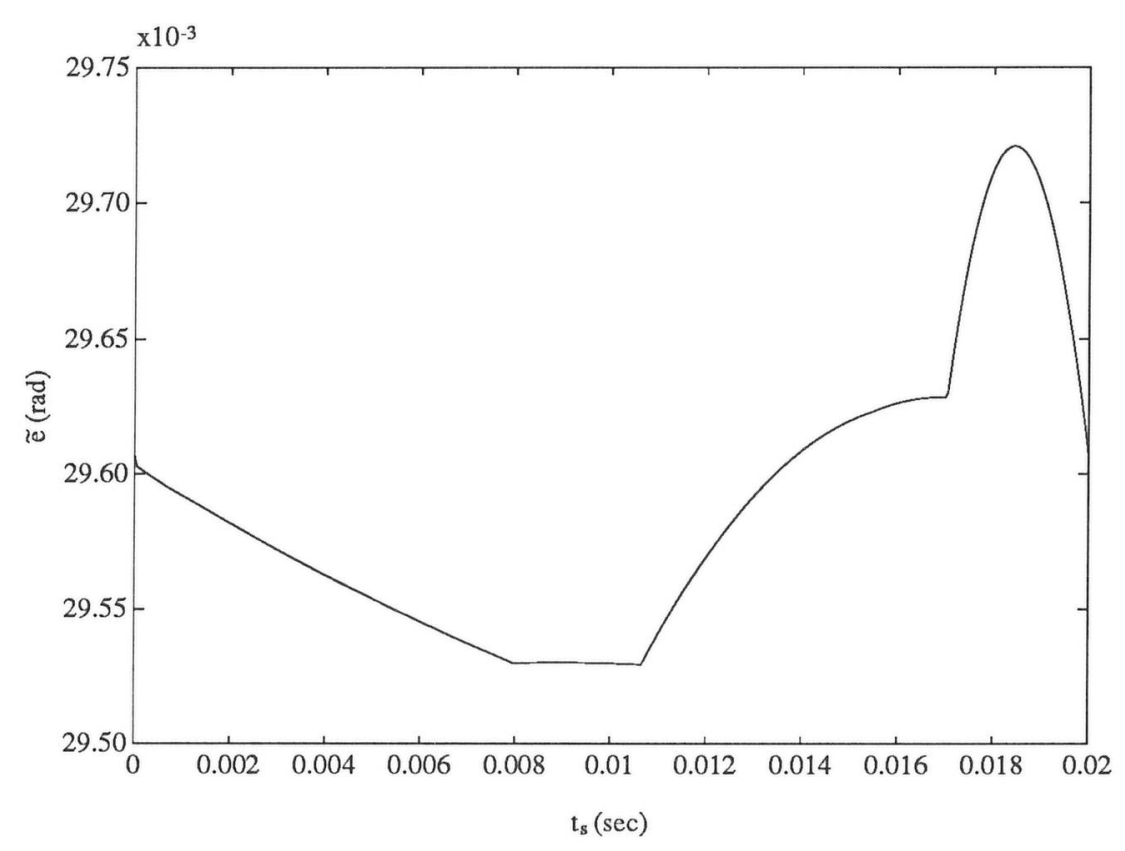

Figure 8: $\tilde{e}\left(t_{s}\right)$ against $t_{s}$ for $T=20 \mathrm{msec}$ 


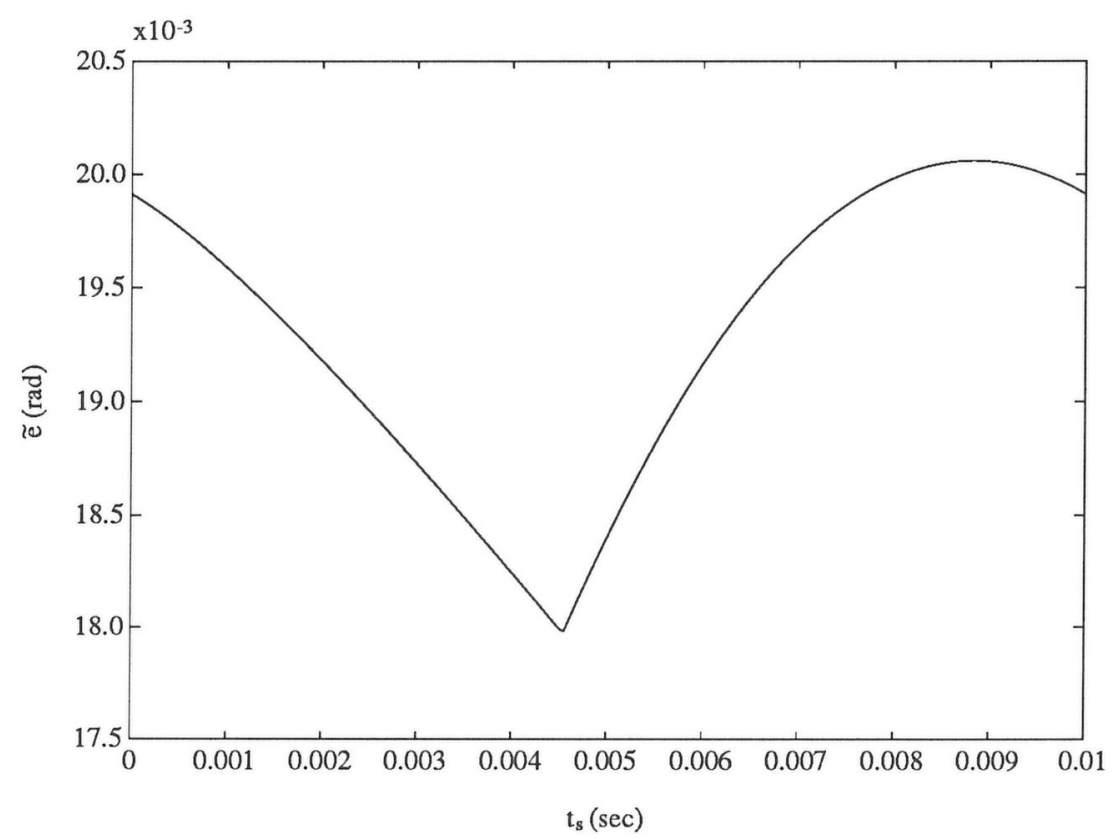

Figure 9: $\tilde{e}\left(t_{s}\right)$ against $t_{s}$ for $T=10 \mathrm{msec}$ 


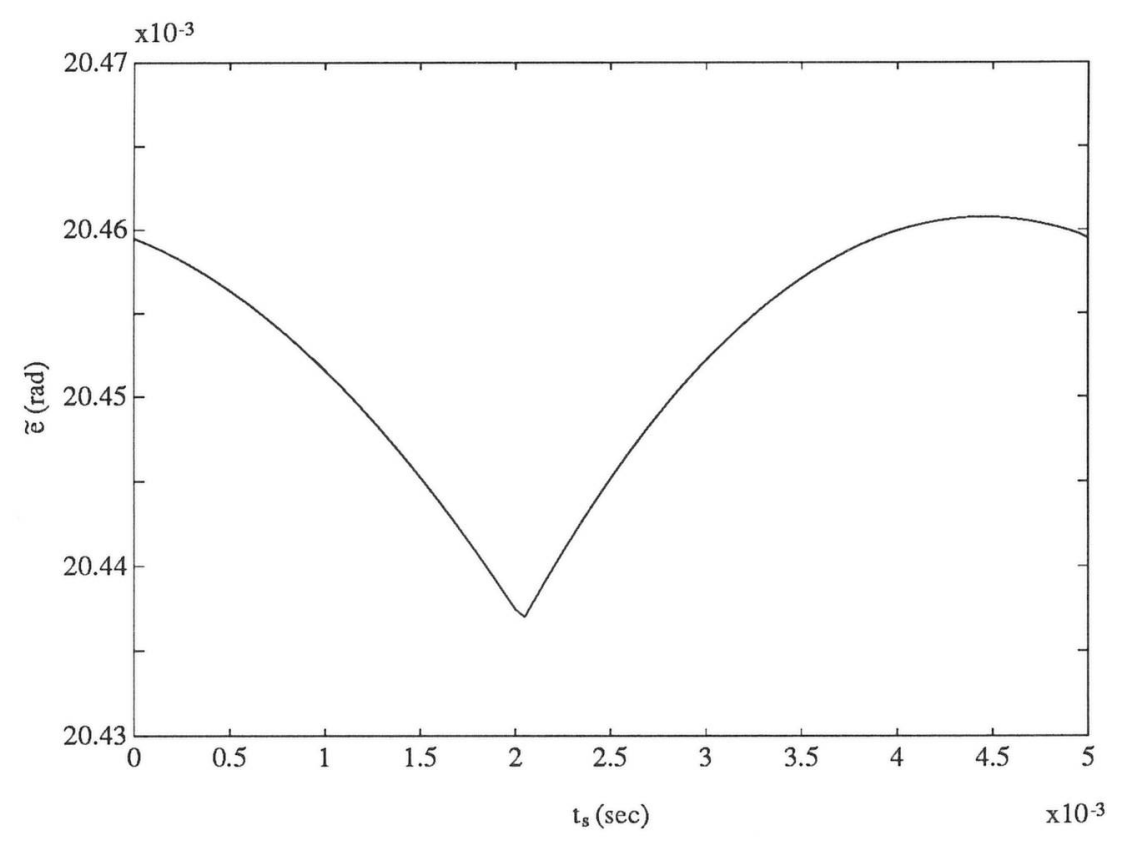

Figure 10: $\tilde{e}\left(t_{s}\right)$ against $t_{s}$ for $T=5 \mathrm{msec}$ 


\section{Cranfield University}

\section{CERES https://dspace.lib.cranfield.ac.uk}

1992-05-01

\section{Performance in sampled data systems}

\section{Whidborne, James F.}

\section{IEEE}

Whidborne, J. F. (1992) Performance in sampled data systems, lee Proceedings D: Control

Theory and Applications, Vol. 139, Iss. 3, pp. 245-250

http://ieeexplore.ieee.org/xpl/articleDetails.jsp?arnumber=141512\&newsearch=true\&queryText=Performance\%20in\%20 Downloaded from Cranfield Library Services E-Repository 